Journal of Astronomical Instrumentation, Vol. 6, No. 2 (2017) 1740007 (15 pages)

(C) The Author(s)

DOI: $10.1142 / \mathrm{S} 2251171717400074$

\title{
The Design and Performance of the Gondola Pointing System for the Sunrise II Balloon-Borne Stratospheric Solar Observatory
}

\author{
A. Lecinski ${ }^{1,3}$, G. Card $^{1}$, M. Knölker ${ }^{1}$ and B. Hardy ${ }^{2,4}$ \\ ${ }^{1}$ High Altitude Observatory \\ National Center for Atmospheric Research \\ Boulder, CO 80307, USA \\ ${ }^{2}$ Dynamics Analysis \& Control \\ LLC, Longmont, CO 80503, USA \\ 3 alice@ucar.edu \\ ${ }^{4}$ bmh5280@gmail.com \\ Received 2016 November 7; Accepted 2017 April 12; Published 2017 May 15
}

\begin{abstract}
With its $1 \mathrm{~m}$ aperture, the Sunrise Balloon-Borne Stratospheric Solar Observatory was the largest spacebased solar telescope. It was designed to study the magneto-convective processes of the sun at resolutions higher than $100 \mathrm{~km}$ and the payload took data during a flight from June 12 to June 17, 2013. To achieve its science requirements, the telescope had to point to an accuracy of $26^{\prime \prime}$ for extended periods of time. Pointing of the instrument was effected by the Sunrise Pointing System (PS). The PS used measurements provided by a Lockheed Intermediate Sun Sensor (LISS) and passed the data through a cascade of up to four digital filters to calculate the best voltages to drive the azimuthal and elevation motors. All filter settings could be modified in flight to adapt to changing conditions. Using this design, the PS met its requirements, pointing the instrument with an accuracy better than $26^{\prime \prime}$ for $60 \%$ of the flight and for continuous time periods of up to $99 \mathrm{~min}$. In this paper, we detail the design and performance of the PS during the 2013 flight.
\end{abstract}

Keyword: Balloons.

\section{Introduction}

The second science flight of the Sunrise BalloonBorne Stratospheric Solar Observatory took place in June of 2013. The stratospheric balloon flight began from ESRANGE (near Kiruna, Sweden) and the payload floated to Northern Canada's Boothia Peninsula. By operating at $36 \mathrm{~km}$ above most of the Earth's atmosphere, the Sunrise telescope was able to collect data in far ultra violet wavelengths (as low as $214 \mathrm{~nm}$ ) and observe the sun without the detriment of atmospheric seeing.

The Sunrise Balloon-Borne Stratospheric Solar Observatory has been fully described by Barthol et al. (2010). The Sunrise Pointing System (PS) was developed by the High Altitude Observatory (HAO) and served multiple purposes, including off-pointing, flat fielding and engineering data collection. Its most critical function was to "coarsely" point the gondola and telescope to a target on the sun with an accuracy better than $26^{\prime \prime}$. Once this pointing accuracy was achieved, the Image Stabilization and Light Distribution (ISLiD) system and the Correlating Wave-Front Sensor (CWS) could deliver a stabilized image to the science instruments with a precision of $0.04^{\prime \prime}$ (RMS). The science instruments were the Sunrise Filter Imager (SuFI) and the

This is an Open Access article published by World Scientific Publishing Company. It is distributed under the terms of the Creative Commons Attribution 4.0 (CC-BY) License. Further distribution of this work is permitted, provided the original work is properly cited. 
Imaging Magnetograph eXperiment (IMaX). Descriptions of ISLiD and SuFI are given in Gandorfer et al. (2011). CWS is described by Berkefeld et al. (2011). Information for IMaX is given by Martínez Pillet et al. (2011).

Pointing a $1920 \mathrm{~kg}$ balloon-borne telescope accurately and continuously towards the sun was a nontrivial matter as there was no fixed structure that could be used to determine the attitude or to react against to orient the telescope. The PS consisted of sensors, actuators and control algorithms that autonomously oriented the telescope. The Sunrise double azimuth control was based in part on the BLAST experiment described by Pascale et al. (2008). The PS was built upon the heritage from the 2007 Sunrise test flight and 2009 Sunrise Science flight but included improvements that are described within. In this paper, we first describe the design of the PS and then use flight data to assess its performance.

\section{Design}

In this section, we review the design of the PS. We first review the actuators and the sun sensors of the instrument, and then provide an overview of the PS program. We then focus on the two most important threads within the PS program and provide details of the pointing algorithms.

A basic block diagram is given in Fig. 1. The PS actuators were: coarse azimuth motor, fine azimuth motor (reaction wheel) and elevation motor. The PS sun sensors were: corner cells (azimuthal detectors), "Precision Azimuth Sun Sensor" (PASS), "Full Range Elevation Detector" (FRED) and Lockheed

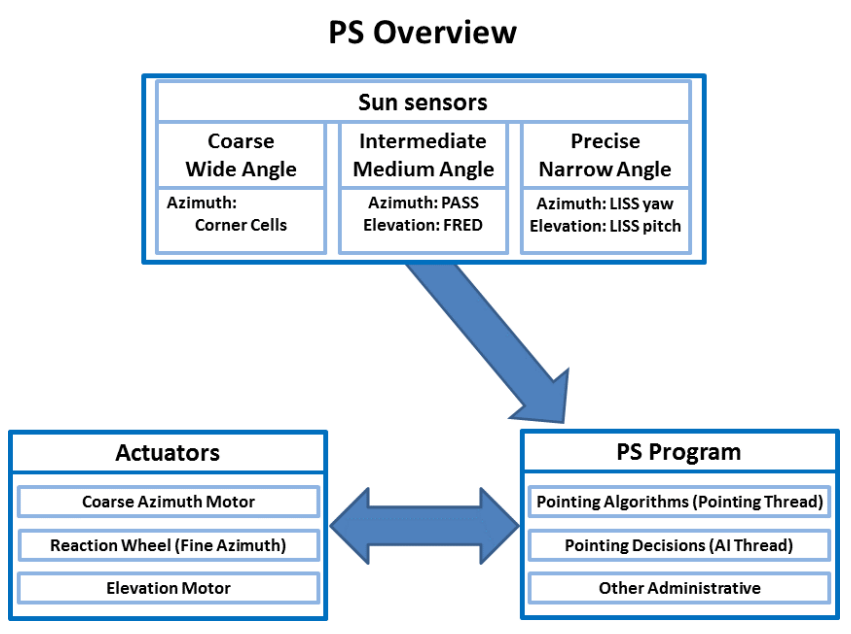

Fig. 1. Overview of PS design.
Intermediate Sun Sensor (LISS yaw and LISS pitch).

\subsection{Pointing system actuators}

A schematic diagram of the Sunrise gondola is shown in Fig. 2. A labeled photo of the Sunrise gondola is shown in Fig. 3. The gondola/telescope could point in both azimuth and elevation. The azimuth drive consisted of a coarse azimuth motor that torqued against the flight train and the balloon, as well as a reaction wheel driven by a separate motor that exchanged angular momentum with the gondola and provided finer tuning of the azimuth motion. Note that the reaction wheel on its own could not provide the azimuth scanning on long time scales as external torques would eventually saturate the speed of the reaction wheel. The specifications of the motors and the reaction wheel can be found in Table 1.

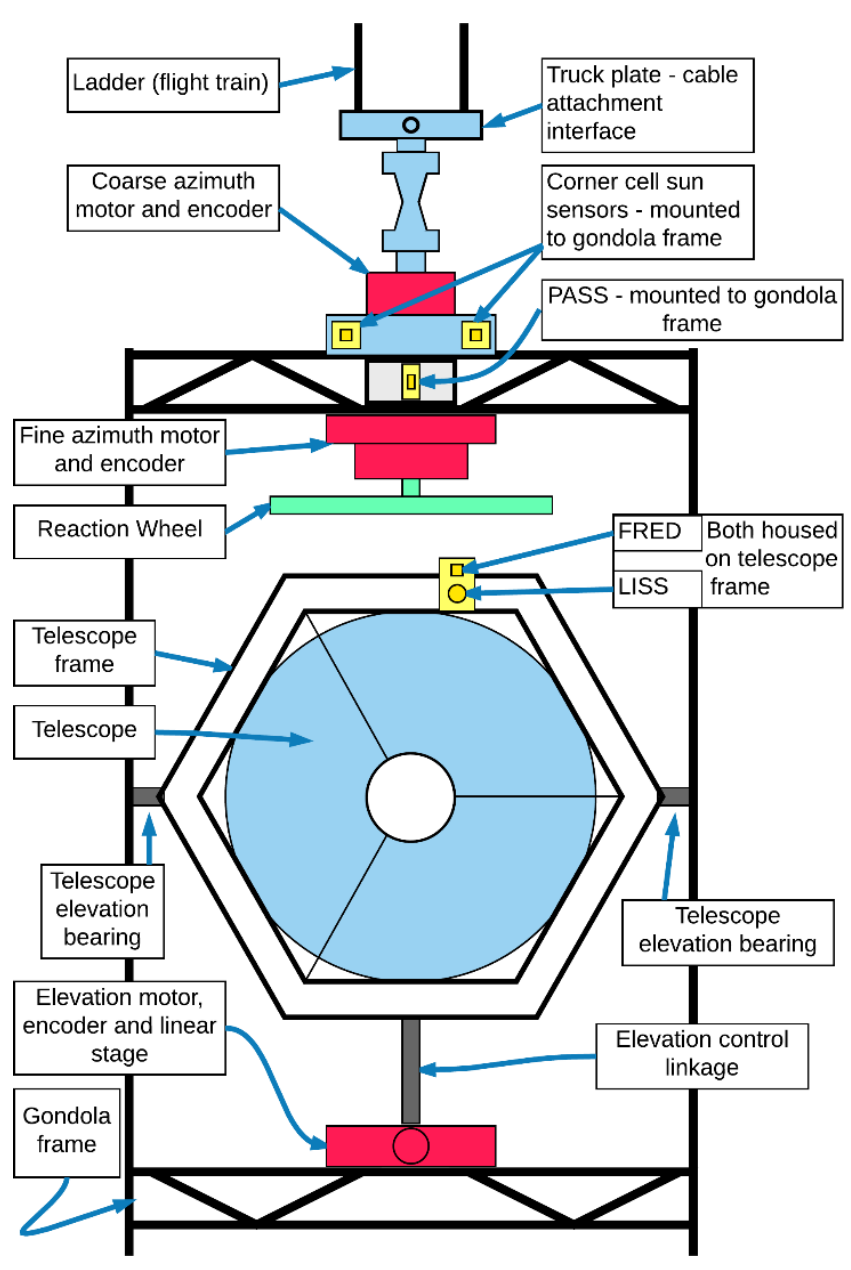

Fig. 2. Schematic diagram of the Sunrise II gondola. 


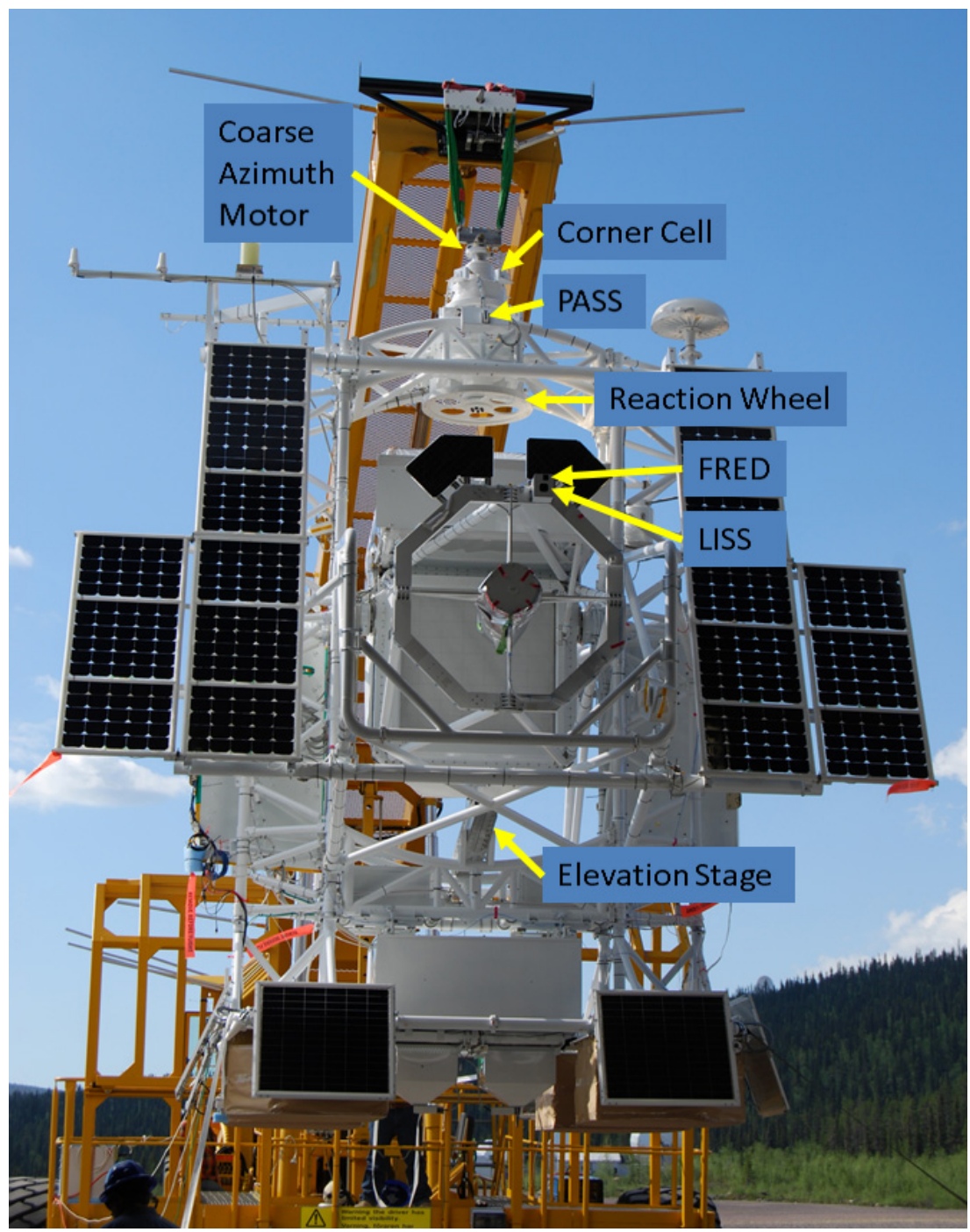

Fig. 3. Labeled photograph of the Sunrise II gondola. The gondola is shown hanging from the Hercules launch vehicle a few days prior to the 2013 launch from ESRANGE near Kiruna, Sweden.

For pointing in elevation, the Sunrise design used an inclined linear stage and a single motor to raise and lower the telescope. The specifications of the motor and the stage are also found in Table 1.

\subsection{Sun sensors}

Multiple sun sensors of various accuracy and viewing angles were placed on the instrument. To be able to first find the sun, the PS used less accurate sun sensors but with wide angle acquisition ranges: the corner cells, PASS and FRED. Once the instrument was roughly pointing at the sun, the more accurate LISS sensor data were used. The specifications for the sun sensors are given in Table 2. Descriptions of their design and uses are given below.
The corner cells were only used during initial sun acquisition pointing. The corner cells were large area silicon photo diodes. Each photo diode was calibrated to produce identical output for a given photon input. Thus, if the front two corner cells had identical output, the gondola was pointing toward the sun. Due to their low accuracy, they only roughly determined the sun's position in azimuth. When the corner cell data were used for tracking, the PS was in low resolution pointing mode.

When the gondola was azimuthally pointed to within a few degrees of the sun, the sun was within the PASS sensor and FRED sensor capture ranges. When the PASS or FRED data were used for tracking, the PS was in intermediate resolution pointing mode. 
Table 1. Specifications for Sunrise II actuators.

\begin{tabular}{lll}
\hline Coarse azimuth & Fine azimuth and reaction wheel & Elevation and linear stage \\
\hline Motor: & Motor: & Motor: \\
Aeroflex & Aeroflex & Aeroflex \\
Catalog \#: Z-1250-200-3-XXX & Catalog \#: Z-1250-200-3-XXX & Catalog \#: Z-0350-300-3-XXX \\
Part \#: 16330 & Part \#: 16330 & Part \#: 16331 \\
Encoder: & Encoder: & Encoder: \\
Heidenhain & Heidenhain & Heidenhain \\
Catalog \#: RON 886 36000 & Catalog \#: RON 886 36000 & Catalog \#: RON 285 18000 01-03 \\
Part \#: 355 886-01 & Part \#: 355 886-01 & Part \#: 358 699-04 \\
Amplifier: & Amplifier: & Amplifier: \\
Copley Controls & Copley Controls & Copley Controls \\
Catalog \#: R20-055-18-S & Catalog \#: R20-055-18-S & Catalog \#: R20-055-18-S \\
& Reaction Wheel: & Linear Stage: \\
& 0.8 meter diameter & Parker \\
& $6061-T 6$ Aluminum & Catalog \#: 406 1500 XRMS D2 H3 L3 C1M3E1B1R1P1 \\
& MOI of 2.865 kg*m ${ }^{2}$ & \\
\hline
\end{tabular}

Table 2. Specifications for Sunrise Sun Sensors.

\begin{tabular}{|c|c|c|c|c|c|}
\hline Sun sensor & $\begin{array}{l}\text { Location and orientation } \\
\text { on gondola }\end{array}$ & $\begin{array}{l}\text { Capture range/ } \\
\text { field of view }\end{array}$ & Linear range & Accuracy & Manufacturer \\
\hline Corner cells & $\begin{array}{l}\text { Far upper gondola frame, } \\
\text { oriented to the four corners } \\
\text { of the gondola. }\end{array}$ & $\begin{array}{l}105^{\circ} \text { in azimuth } 60^{\circ} \text { in } \\
\text { elevation }\end{array}$ & $\begin{array}{l}\text { Same as capture } \\
\text { range. }\end{array}$ & $\sim 1$ degree & $\mathrm{HAO}$ \\
\hline PASS & $\begin{array}{l}\text { Upper gondola frame, facing } \\
\text { forward. }\end{array}$ & $\begin{array}{l} \pm 3^{\circ} \text { in azimuth } \pm 90^{\circ} \text { in } \\
\quad \text { elevation }\end{array}$ & $\pm 3^{\circ}$ in azimuth & $\sim 10^{\prime \prime}$ & $\mathrm{HAO}$ \\
\hline FRED & $\begin{array}{l}\text { Upper telescope frame, facing } \\
\text { forward. }\end{array}$ & $\begin{array}{l} \pm 5^{\circ} \text { in azimuth } \pm 60^{\circ} \text { in } \\
\quad \text { elevation }\end{array}$ & $\pm 15^{\circ}$ in elevation & A few arcminutes & $\mathrm{HAO}$ \\
\hline LISS & $\begin{array}{l}\text { Upper telescope frame, facing } \\
\text { forward. }\end{array}$ & $\begin{array}{l} \pm 3^{\circ} \text { in azimuth and } \\
\text { elevation }\end{array}$ & $\begin{array}{l} \pm 15^{\prime} \text { in azimuth } \\
\text { and elevation }\end{array}$ & $1^{\prime \prime}$ to $2^{\prime \prime}$ & Lockheed \\
\hline
\end{tabular}

The PASS was a shadow type sun sensor. The PASS design had two large area silicon photo diodes placed side by side in the azimuth direction and between the diodes was a very thin shadow casting strip. When the sun was directly in front of the PASS, no shadow was cast and the diodes produced identical output. The two diodes were wired together to produce a pointing error signal that was the difference of the output between the two diodes. A zero error signal indicated good pointing. Unfortunately, the error signal was also zero when the when the sun was located beyond the bounds of the PASS capture range. To insure the PASS pointing error data were only used when the sun was within the PASS capture range, the PASS design also included a separate photo diode to measure incoming solar intensity. A large intensity signal coming from this photo diode was used to confirm the validity of the PASS pointing error data. We called this intensity signal the PASS Sun Present signal.

The FRED sensor consisted of two large area silicon photo diodes arranged on an A-frame structure rotated by $90^{\circ}$ such that the apex pointed in the elevation direction. The diodes on each side of the A-frame were wired together to produce a pointing error signal that was the difference of the output between the two diodes. A positive or negative signal indicated the sun was above or below the telescope and a zero signal indicated good pointing. Similar to the PASS, the FRED error signal was also unfortunately zero when the sun was beyond the bounds of its capture range. Thus, the FRED also required an indicator to confirm that the sun was within its field of view. Since the FRED and PASS capture ranges were designed to overlap, the 
validity of the FRED pointing error data was confirmed with the PASS Sun Present signal.

When the telescope was pointed to within 15 arcminutes of the sun, the sun was within the capture range of the LISS sensor. The LISS sensor consisted of five photodiodes beneath a square aperture window. The central diode provided a Sun Present signal, the difference in output between the right/left diodes provided an azimuthal (yaw) pointing error signal, and the difference in output between the top bottom diodes an elevation (pitch) pointing error signal. A large LISS Sun Present signal indicated valid data. When the LISS data were used for tracking, the PS was in precise resolution pointing mode. Precise resolution pointing mode achieved the desired pointing accuracy of $\pm 26^{\prime \prime}$.

The Sunrise telescope was able to off-point from sun center by moving the LISS sensor relative to the telescope frame. The LISS was mounted on a two axis motorized tip/tilt stage, which had precise subarc second resolution encoders with a range of $4^{\circ}$. This allowed the telescope to observe features located anywhere on the sun and even beyond the solar limb. It also provided in flight co-alignment between the LISS and the telescope. In flight coalignment capability was desired as launch forces could perturb alignment. Co-alignment was accomplished by moving the LISS stage as CWS looked for the solar limb. Once the location of the north, south, east and west limbs were found, orientation and spatial scale were computed, stored in memory and saved to the PS disk.

The sun sensors produced analog voltages, which were converted to digital units with a Diamond Systems Analog to Digital (A/D) Converter/ Counter Timer board. The digital data from the Diamond board were sampled at $150 \mathrm{~Hz}$ by the PS program's Pointing thread, described in more detail in Sec. 2.5.

\subsection{Pointing system program overview}

The PS computer, a Diamond systems PLTN270XT-2G, ran the highly optimized PS program, which was written in $\mathrm{C}++$. In addition to pointing, the PS program had to simultaneously perform other administrative tasks, and hence was multithreaded. Threads consisted of:

- Artificial Intelligence (AI) thread: The role of this thread was to autonomously orchestrate PS pointing. The thread made pointing mode decisions and smoothed the transition from one pointing mode to the next pointing mode. In order to compile long term statistical information from the sun sensors and motors, this thread ran relatively slowly, once every $9 \mathrm{~s}$.

- Pointing thread: This thread performed the crucial role of pointing the gondola and telescope. To insure the PS achieved the desired pointing accuracy of better than $\pm 26^{\prime \prime}$, this thread ran quickly at $150 \mathrm{~Hz}$.

- Incoming command thread: This thread processed incoming commands from the Instrument Control Computer (ICU). This thread only ran when a new ICU command was received.

- CWS thread: This thread processed incoming commands from the CWS. This thread only ran when a new CWS command was received.

- CWS PS pointing lock thread: This role of this thread was to immediately signal the CWS whenever the PS pointing was within the capture range of the CWS components, $\pm 6^{\prime \prime}$.

- Write data thread: This thread wrote all PS data to the ICU. To insure no data was lost, this thread ran at a slightly faster rate than memory buffers were filled (every $5 \mathrm{~s}$ ).

- House Keeping thread: The role of this thread was to regularly transfer a small snippet of the most recent PS data to ground personnel. With this data ground personnel were able to roughly assess PS performance and health. To keep ground personnel frequently updated, this thread ran at $1 \mathrm{~Hz}$.

- Thumbnail thread: The role of this thread was to provide detailed sun sensor and motor data in a "thumbnail" packet to ground personnel. With the thumbnail data, ground personnel were able to determine how well pointing algorithms were behaving and adjust parameters as needed. Thumbnail data proved pivotal in fine tuning parameters and improving pointing accuracy. As bandwidth was limited, thumbnails were only sent to ground when pointing improvements were required.

The workhorses of the PS program were the AI thread and the Pointing thread. They are described in detail in Secs. 2.4 and 2.5.

\subsection{AI thread}

The AI thread had two main goals: to decide which pointing mode to place the telescope in and to find 
the pointing alignment offsets between the various sensors. This thread allowed the PS to autonomously point to the sun from any initial gondola/ telescope position.

In order to transition from a disoriented pointing to a highly accurate pointing, the PS had three pointing modes called low, intermediate and precise. Azimuth and elevation axes were treated independently. Each mode used different sensors and algorithms to orient the telescope. The instrument initially started in low resolution pointing mode and the PS slowly rotated the gondola toward the sun. Once the AI thread had determined that the gondola was stably pointing to the sun within a few degrees in azimuth, intermediate azimuthal pointing mode would begin. Simultaneously, sunlight became available on the elevation sensors, and the AI thread then activated the intermediate elevation pointing mode. When the AI thread determined that azimuthal or elevation pointing was stable to within a few arcminutes, it activated the precise (arc second) pointing mode for that axis. Additionally, steps could be skipped so that the precise pointing mode could start immediately as long as relevant criteria were met.

The AI thread used running means and running standard deviations of the sun sensors, motor voltages and motor velocities to determine if a pointing mode was stable. These statistics were also used to determine if a higher resolution pointing mode could be enabled. Each pointing mode had an associated set of variables to enable/disable certain sensors and certain pointing algorithms. For example, for the low azimuthal pointing mode, only the data from corner cell sun sensors were enabled, and only the pointing algorithm for the coarse azimuth motor (see details in the following section) was enabled.

Another major role of the AI thread was to calibrate the alignment angles between each sensor. In order to compute the misalignment angles, the AI thread would progressively add a small numerical offset to a sensor reading until the statistics of the next most accurate sensor improved. If the statistics of the next sensor did not improve, the AI thread would try adding an offset in the opposite direction. Once a good offset was found, it was applied to the coarser sensor, retained in memory and saved to a file on the PS disk. In this manner the PS calculated the alignments between the corner cells and the PASS, the corner cells and the LISS yaw, and the FRED and the LISS pitch. Thus if any misalignment between sun sensors existed or changed at launch or occurred during the flight, it was automatically handled by the AI thread. The offsets could be set via commands from ground. Similarly, threshold settings for statistics and pointing offsets could be modified in flight via commands sent from ground. Auto-pointing could be commanded to be enabled or disabled and commanded to go into manual mode.

\subsection{Pointing thread}

The primary role of this thread was to point the gondola and telescope by continually adjusting motor voltages to minimize pointing errors seen by the sun sensors. Additional roles were to sample environmental data and store all data in memory buffers.

The Pointing thread was called each time an I/O interrupt was generated by the Diamond Systems Analog to Digital (A/D) Converter/Counter Timer board, model DSC_DMM32DXAT. The thread first captured the current sample of all of the data provided by the $\mathrm{A} / \mathrm{D}$ board: sun sensor data, amplifier and motor temperatures, PS computer environmental data, and three axis accelerometer data from two separate areas on the gondola and one on the telescope. The thread next queried the coarse azimuth, fine azimuth and elevation encoders for current positions. The thread then distributed the sun sensor data, reaction wheel velocity and elevation motor velocity through the pointing algorithms. The output of the algorithms produced voltages that the thread sent to the appropriate azimuthal or elevation motor to improve pointing. The pointing algorithms are described in the next section.

The rate at which the Pointing thread was called had to be set to be fast enough to achieve the desired pointing consistency and accuracy. The lowest rate needed to achieve the desired pointing accuracy was calculated to be $\sim 100 \mathrm{~Hz}$. Faster rates yielded improved performance. Code optimization allowed the PS Pointing thread to run quickly, so the Diamond Counter/Timer board was set to run at $150 \mathrm{~Hz}$.

The Pointing thread was highly optimized for speed and efficiency. It was coded to insure that it consumed identical clock times and identical CPU cycles every time it was trigged for execution. No "if" or "case" statements were used. Instead, all calculations for all light sensors and all pointing 
algorithms were computed in each sample. The outputs of the algorithms were multiplied by the disable/enable values set by the AI thread, and then were added all together and sent to the appropriate motors. Since the Pointing thread just did sampling and numerical calculations, and never had to choose what light sensor to use or what algorithm to use, the thread always ran in the same amount of time. The CPU cycles used by the Pointing thread easily fitted within $150 \mathrm{~Hz}$.

\subsection{Pointing algorithms}

The pointing algorithms were "servo suites" that took as input sensor and motor data to compute the necessary voltages for the azimuth and elevation motors in order to orient the telescope to the desired attitude. They were composed of up to four sequential user selectable digital filters (low pass, high pass, lead, lag, integrator, notch, peak or one-toone). The output from the filters was multiplied by a servo suite gain.

Each pointing mode had its own set of servo suites. The AzcTrack servo suite implemented low resolution pointing mode. The AzfTrack and AzfToAzc servo suites implemented intermediate and precise azimuthal pointing modes. And the
El_Track, El_Local and El_Frict function implemented the intermediate and precise elevation pointing modes. A block diagram showing the servo suites used in precise pointing mode is shown in Fig. 4.

The details for each servo suite follow:

- AzcTrack: Implemented low resolution azimuth pointing mode using corner cell data for input. The output from AzcTrack suite was a voltage to the Azc (coarse azimuth) motor, which rotated the entire gondola.

- AzfTrack: Implemented intermediate azimuthal pointing mode when using the PASS signal as input, or precise azimuth pointing mode when using the LISS yaw as input. The output from the AzfTrack suite was a voltage to the Azf motor, which accelerated or decelerated the reaction wheel. The acceleration/deceleration of the reaction wheel rotated the gondola by small, precise amounts. A desired Azf velocity of 10 RPM ensured that the Azf motor was nearly always in motion.

- AzfToAzc: Spun off excessive Azf (reaction wheel) velocity to Azc. The input was the difference between the desired Azf velocity and the actual Azf velocity.

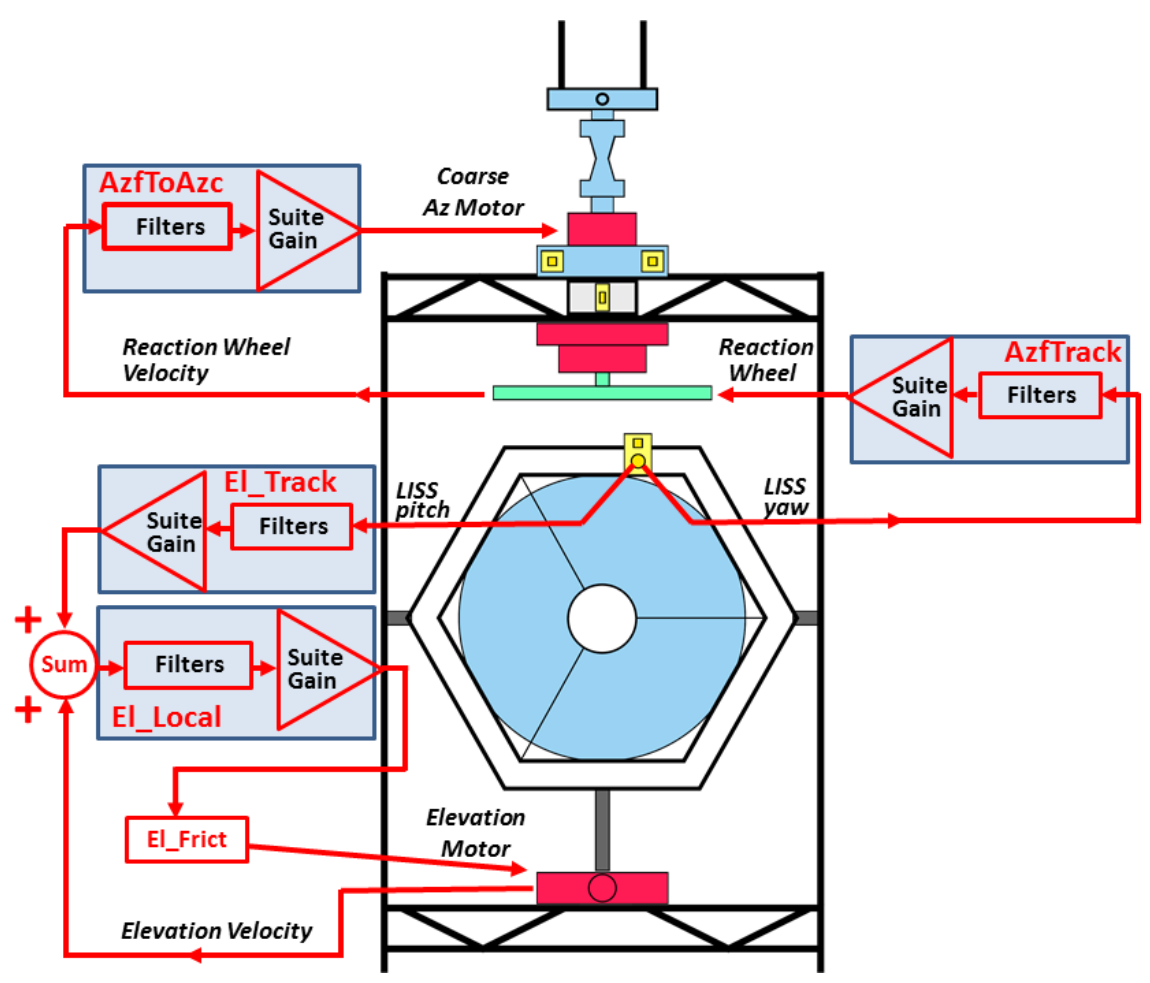

Fig. 4. Block diagram showing the servo suites used in precise pointing mode. 
The output from the AzfToAzc suite was a voltage to the Azc motor, which very slightly rotated the entire gondola. This very slight rotation allowed the reaction wheel to slow.

- El_Track: Implemented intermediate elevation pointing mode when using the FRED signal as input, or precise elevation pointing mode when using the LISS pitch signal as input. The results from the El_Track suite were passed to the El_Local servo suite.

- El_Local: Acted to reduce the effects of bearing friction and changed the output of $\mathrm{El}_{-}$Track from a motor current (the effects of which will change with temperature) to a calibrated velocity. The results from the El_Local suite were passed to the El_Frict function.

- El_Frict: Friction was reduced further with the Friction Compensation function. This was not a digital filter but rather a function that compensated for stiction in the motor/drive as well as gravitational effects. It is detailed below. The output from this function was the voltage applied to the El_(elevation) motor, which then drove the telescope lever arm along the inclined elevation stage. Movement of the lever arm raised or lowered the telescope.

With the exception of the El_Frict function, the filters used in the above servo suites were all cascaded digital filters. The digital filters used by the PS followed the form:

$$
y[n]=-\sum_{k=1}^{M} a_{k} y[n-k]+\sum_{k=0}^{N} b_{k} x[n-k] .
$$

The type of filter, low pass, lead, notch, etc., determined the $a_{k}$ and $b_{k}$ coefficients. The PS used both 1 st order $(M=1$ and $N=1)$, and 2 nd order $(M=2$ and $N=2)$ filters.

Filters for each suite were chosen based on models of gondola structure, its resonances, its pendulum modes and also on each motor's characteristics. Settings were refined with data acquired during hang tests where white noise was added to a servo input and the servo output was split out to a HP 35660A Digital Signal Analyzer.

Selection of the optimal suite gains and filter frequencies ensured that a servo remained stable, resonances of the mechanical structure of the gondola were not excited, and pendulum motions were not reinforced. Vibrations of the telescope were particularly undesirable as they negatively affected the CWS performance. High frequency vibrations prevented the CWS from achieving a closed loop control lock of the image stabilization system, and low frequency vibrations could create residual image smear.

Settings for all of the servos and functions could be modified in-flight via commands sent through the ICU. Modification of the settings allowed the PS to improve pointing performance and adapt to nuances of evolving flight conditions. Thumbnails containing $6 \mathrm{~s}$ of $150 \mathrm{~Hz}$ sun sensor data, motor voltages and velocities provided detailed servo performance information. Analysis on the ground by servo engineers determined where improvements were needed in the servo settings. Improved settings could quickly be sent up to the PS and activated. The settings in Table 3 produced a continuous $99 \mathrm{~min}$ period during which pointing was within $\pm 26^{\prime \prime}$ of the target. The low pass filters removed high frequency noise. The lead filters effected a phase lead which enhanced responsiveness. The high quality notch filters were tuned to suppress gondola pendulum frequencies and elevation motor response frequencies. The integrators provided high pointing accuracy.

Suite gains also had to be chosen with care. The deleterious effect of too large a suite gain is shown in data from the commissioning phase of the mission. During the commissioning phase, servo settings similar to those of the 2009 flight were initially tried and subsequently adjusted. A trial setting applied on 2013 June 12 at 10:08:24 used fairly high servo suite gain for AzfTrack (-400.). As seen in Fig. 5, this resulted in a very high noise level, particularly at a gondola structural resonance at $10 \mathrm{~Hz}$. A downloaded thumbnail revealed the problem to the PS engineers, who determined a smaller AzfTrack servo suite gain was required. At 10:08:38 an AzfTrack servo suite gain of -150.0 was uploaded to the PS, and as seen in Fig. 6, the smaller gain resulted in greatly improved pointing and much less noise at $10 \mathrm{~Hz}$.

The El_Frict friction compensation function was an improvement added for the 2013 flight. It compensated for stiction in the motor/drive and varying gravitational effects along the inclined elevation stage. For most motors, a nonlinear friction region exists where it is more difficult to initiate the rotation of the axle. In this area, more force, hence higher voltage must be used. If one does not 
Table 3. Servo settings for the 2013 Sunrise flight. Settings shown produced a 99 min period where pointing was continuously within $\pm 26^{\prime \prime}$.

\begin{tabular}{|l|l|l|}
\hline \hline \multicolumn{2}{|l|}{ AzfTrack settings: } & suite gain -150.00 \\
\hline Order & Type & Settings \\
\hline 2nd & Low pass & Frequency $=5 . \quad$ Quality $=1$. \\
\hline 1st & Lead & Zero $=0.1 \quad$ Pole $=20$. \\
\hline 1 st & Integrator & Frequency $=0.11$ \\
\hline
\end{tabular}

\begin{tabular}{|c|c|c|}
\hline \multicolumn{2}{|c|}{ AzfToAzc settings: } & suite gain $\quad-900.00$ \\
\hline Order & Type & Settings \\
\hline 1 st & Lead & Zero $=0.1$ \\
\hline 1 st & Integrator & Frequency $=0.005$ \\
\hline
\end{tabular}

\begin{tabular}{|l|l|l|}
\hline \multicolumn{3}{|l|}{ El_Track settings: } \\
\hline Order & Type & Settings \\
\hline 2nd & Low pass & Frequency $=5 . \quad$ Quality $=1$. \\
\hline 1st & Integrator & Frequency $=0.125$ \\
\hline 2nd & Notch & $\begin{array}{l}\text { Frequency }=0.43 \\
\text { Quality }=4.7\end{array}$ \\
\hline 2nd & Notch & Frequency $=5 . \quad$ Quality $=5$. \\
\hline \hline El_Local settings: & suite gain 2.0 \\
\hline Order & Type & Settings \\
\hline 1st & Integrator & Frequency $=100$. \\
\hline 1st & Lead & Zero $=5.0 \quad$ Pole $=20$. \\
\hline
\end{tabular}

\begin{tabular}{|llr|}
\hline El_Frict settings: & El_FrictSlope & 10.0 \\
& El_FrictThreshPlus & 1.2 \\
& El_FrictThreshMinus & -1.2 \\
\hline
\end{tabular}

compensate for this behavior, any small voltage sent within the friction region will not move the motor the desired amount, and the next servo loop will see the increased pointing error and send ever larger voltages to the motor. This typically causes oscillating overshoot/undershoot ("limit cycle") and very poor pointing performance.
To compensate, a friction compensation function can be implemented. Friction compensation functions assume many shapes depending on what details are included in the friction model. For Sunrise, a simple Coulomb friction model was used and was designed to match the specifics of the Sunrise elevation motor and elevation stage
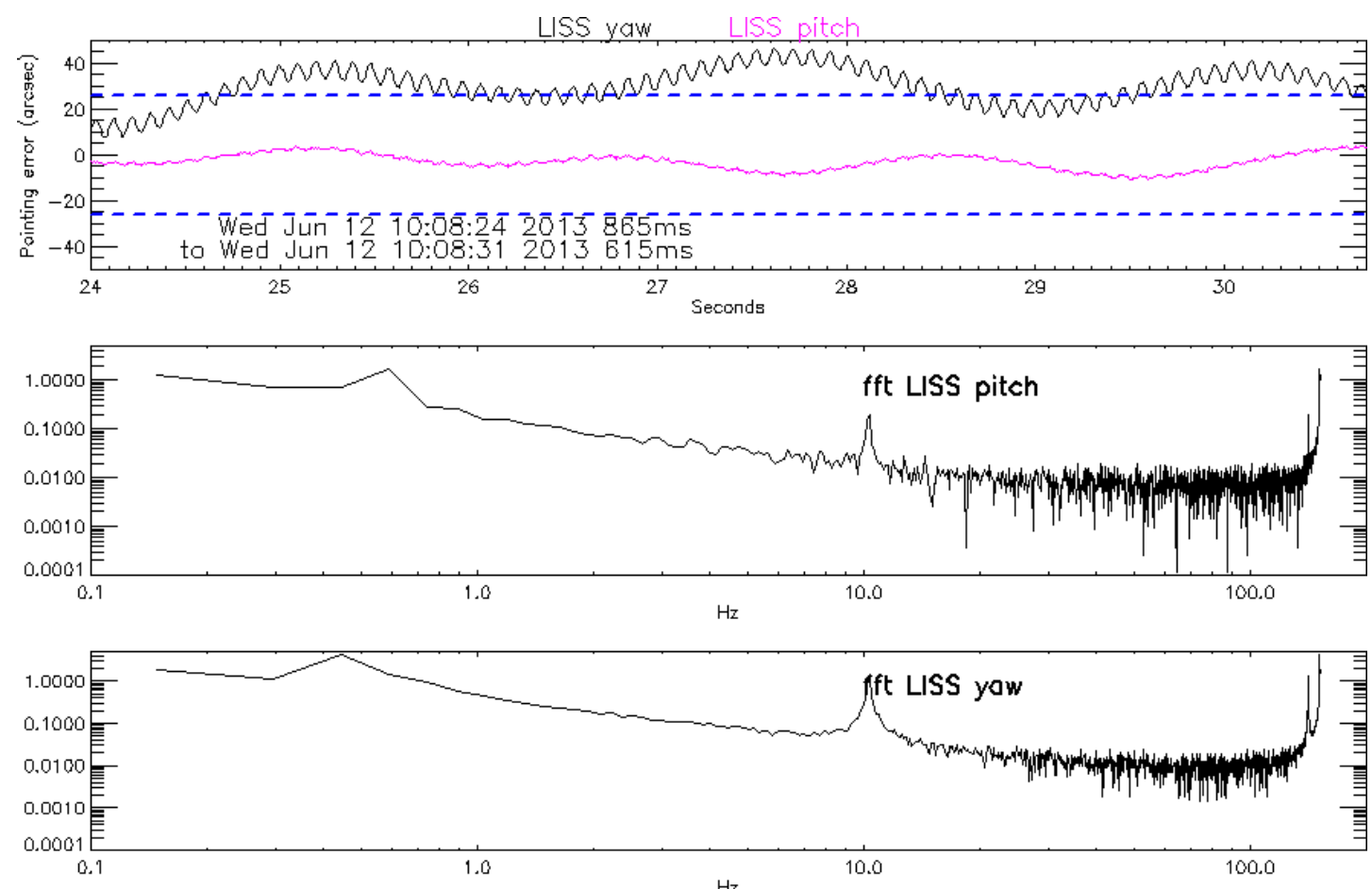

Fig. 5. Plot showing deleterious effects of too large servo suite gain for AzfTrack: a $10 \mathrm{~Hz}$ gondola resonance has been excited. The dashed blue lines in the LISS plot are visual aides showing the boundries of the $\pm 26^{\prime \prime}$ pointing limit. 

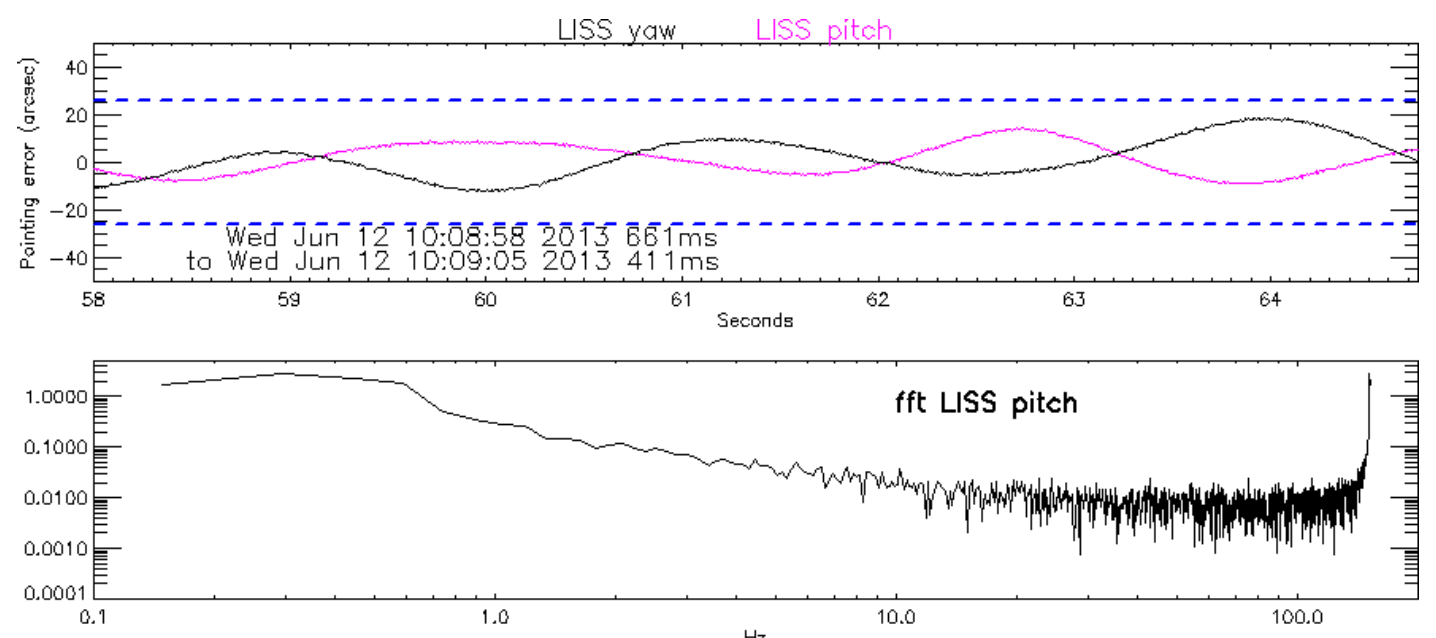

$\mathrm{Hz}$

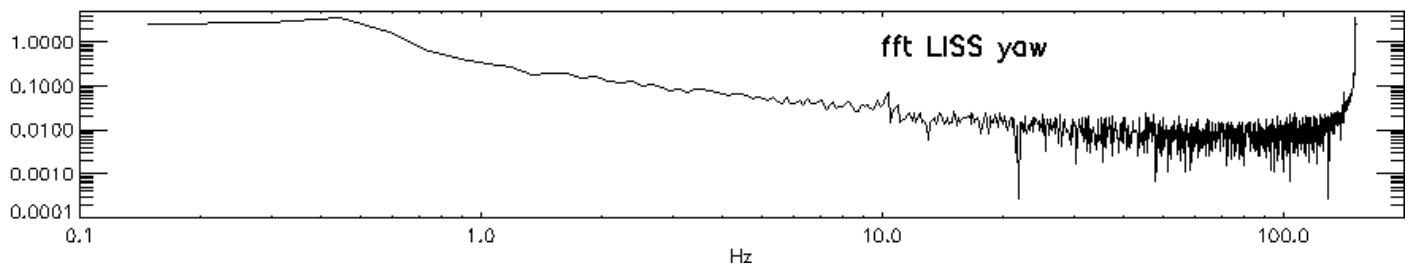

Fig. 6. Reduction of the AzfTrack servo suite gain by a factor $\sim 2.7$ greatly improved pointing and decreased noise, notably the $10 \mathrm{~Hz}$ gondola resonance. The dashed blue lines in the LISS plot are visual aides showing the boundries of the $\pm 26^{\prime \prime}$ pointing limit.

geometry. The Sunrise friction compensation function simply boosted the voltage within a small region around the origin with a multiplicative factor, El_FrictSlope. Outside of the region offsets were added. The equations follow:

$$
\begin{aligned}
& \text { if }\left(V_{\text {in }} \geq \frac{\text { Thresh }_{\text {plus }}}{\text { El_FrictSlope }}\right) \quad V_{\text {out }}=V_{\text {in }}+\text { Thresh }_{\text {plus }} \\
& -\frac{\text { Thresh }_{\text {plus }}}{\text { El_FrictSlope }} \\
& \text { else if } \\
& \left(V_{\text {in }} \leq \frac{\text { Thresh }_{\text {minus }}}{\text { El_FrictSlope }}\right) \quad-\frac{\text { Thresh }_{\text {minus }}}{\text { El_FrictSlope }} \\
& V_{\text {out }}=\text { El_FrictSlope } \times V_{\text {in }} .
\end{aligned}
$$

Figure 7 shows the output voltage as a function of the input voltage for the cases in Eq. (2).

To accommodate evolving conditions, telescope loading, temperature changes or other issues affecting the friction characteristics of the motor, El_FrictSlope, Thresh minus $_{\text {and }}$ Thresh plus $_{\text {could be }}$ individually modified. During the flight only symmetric adjustments of Thresh minus $_{\text {and Thesh }}$ plus were needed to maintain good motor performance and good pointing. These adjustments were likely necessary due to the nonlinearity of the elevation stage design as well as diurnal temperature variations.

As Figs. 8 and 9 indicate, the use of the El_Frict friction compensation function was critical to

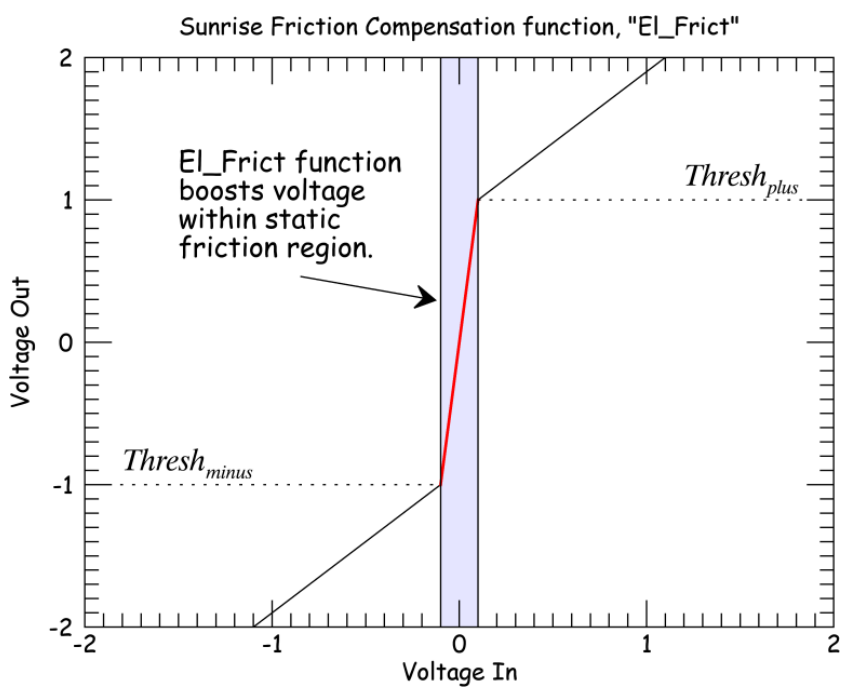

Fig. 7. El_Frict function overcame stiction within the elevation motor. Within the static friction region the voltage was boosted to compensate for the additional force needed to overcome static friction. El_Frict assumed a simple Coulomb friction model, following Eq. (2). In the above example, El_FrictSlope = 10, ThreshMinus $=-1.0$ and ThreshPlus $=+1.0$. 

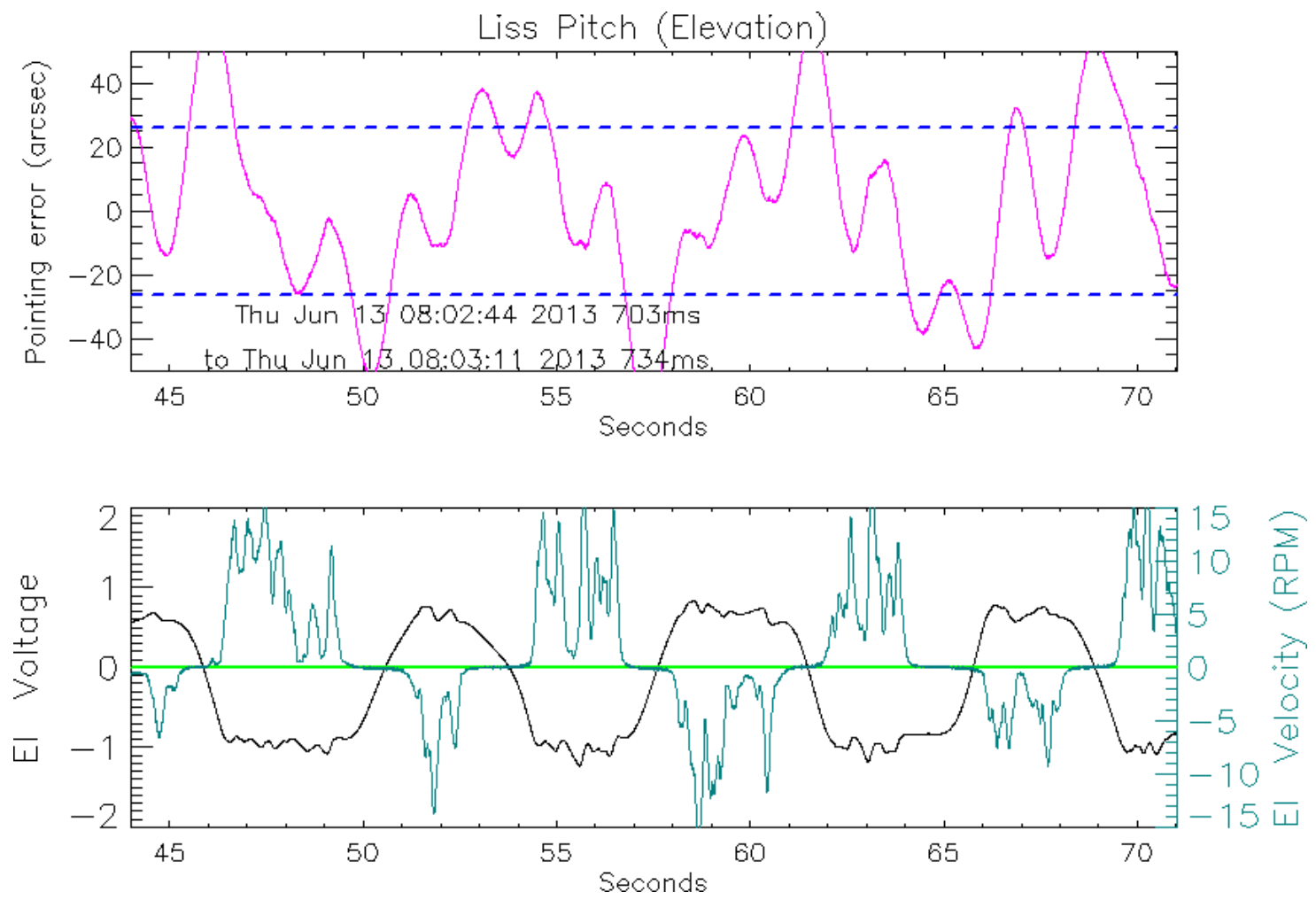

Fig. 8. Friction compensation not in use. Note large pointing errors, voltages and velocity excursions. The dashed blue lines in the LISS plot are visual aides showing the boundries of the $\pm 26^{\prime \prime}$ pointing limit. The bright green line in the Voltage/Velocity plot is a visual aide marking zero voltage and velocity.
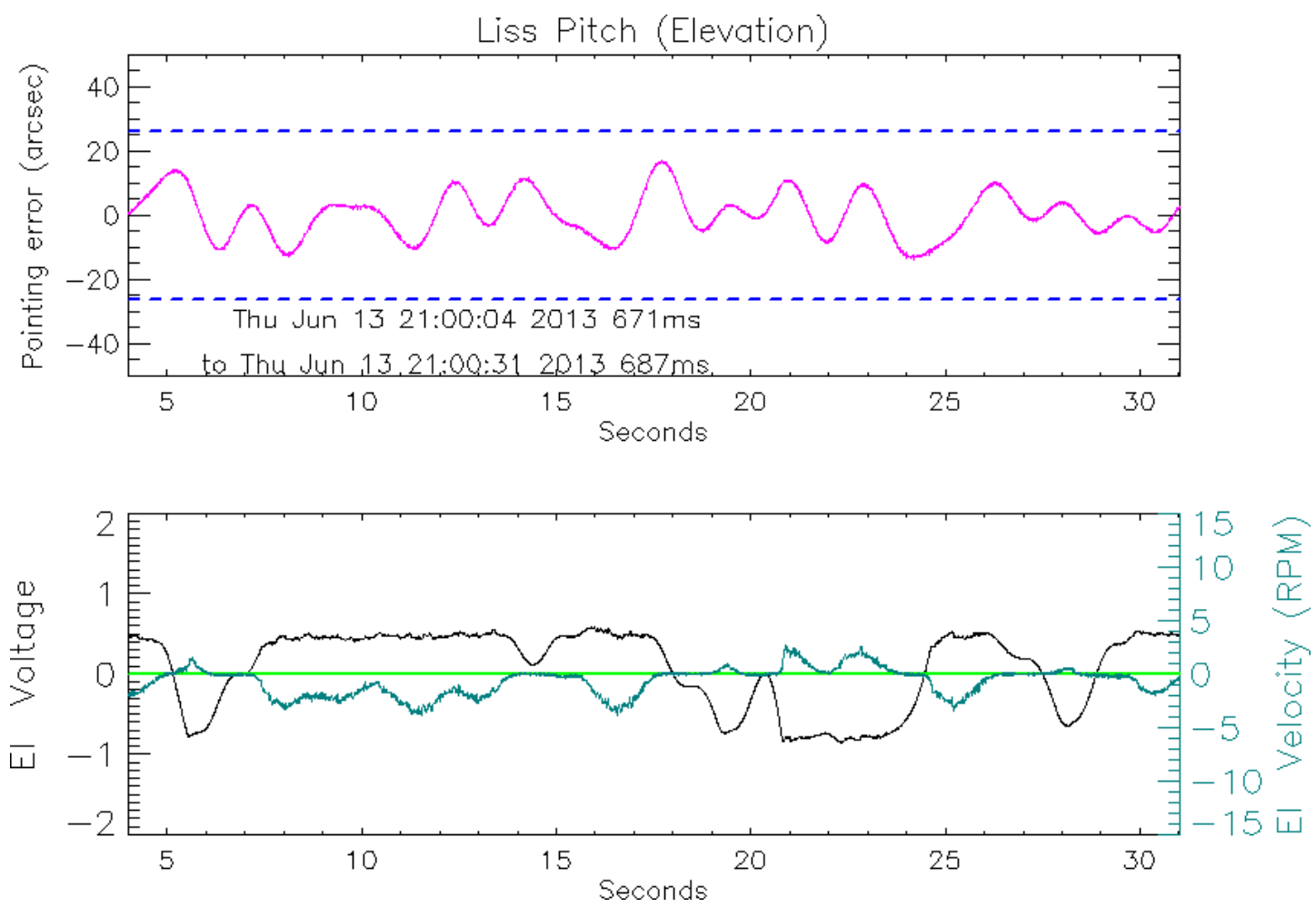

Fig. 9. Friction compensation in use. Pointing has greatly improved. Voltages and velocities no longer over shoot. The dashed blue lines in the LISS plot are visual aides showing the boundries of the $\pm 26^{\prime \prime}$ pointing limit. The bright green line in the Voltage/Velocity plot is a visual aide marking zero voltage and velocity. 
Table 4. Total duration of flight with the gondola/telescope pointing within $\pm 26^{\prime \prime}$ of target. The sum only includes continuous times of a minimum duration.

\begin{tabular}{|c|c|c|c|}
\hline $\begin{array}{l}\text { Minimum continuous time } \\
\text { period with gondola/telescope } \\
\text { pointing within } \pm 26^{\prime \prime} .\end{array}$ & $\begin{array}{c}\text { Sum of time in } \\
2013 \text { (Sunrise II) flight } \\
\text { with gondola/telescope } \\
\text { pointing within } \pm 26^{\prime \prime} . \text { (hours) }\end{array}$ & $\begin{array}{c}\text { Percent of entire } \\
\mathbf{2 0 1 3} \text { (Sunrise II) } \\
\text { flight with gondola/telescope } \\
\text { pointing within } \pm 26^{\prime \prime}\end{array}$ & $\begin{array}{c}\text { Percent of entire } \\
\mathbf{2 0 0 9} \text { (Sunrise I) } \\
\text { flight with gondola/telescope } \\
\text { pointing within } \pm 26^{\prime \prime} .\end{array}$ \\
\hline $30 \mathrm{~s}$ & 80.9 & $67 \%$ & $48 \%$ \\
\hline $1 \mathrm{~min}$ & 72.5 & $60 \%$ & $40 \%$ \\
\hline $2 \mathrm{~min}$ & 62.5 & $52 \%$ & $30 \%$ \\
\hline $10 \mathrm{~min}$ & 33.3 & $28 \%$ & $12 \%$ \\
\hline
\end{tabular}

maintaining good pointing. In Fig. 8, where no friction compensation was in use, pointing was erratic, elevation motor voltage was quite high, and elevation motor velocities varied widely and rapidly. In Fig. 9, where friction compensation was implemented, pointing was greatly improved, motor voltages were smaller and velocities excursions were greatly diminished.

When the friction compensation function was not used, the extra push caused by the servo overshoot could induce pendulum motions. As seen in Fig. 8, the large excursions with a $\sim 8$ s periodicity appear to be due to an overshoot/undershoot induced pendulum motion of the telescope. The smaller excursions with a $\sim 2$ s periodicity exist in both figures. These were likely caused by the short period oscillation of the gondola on its suspension system (flight train).

\section{Results}

During the 2013 flight of $\sim 120 \mathrm{~h}$, the gondola/ telescope was pointing within $\pm 26^{\prime \prime}$ for more than $50 \%$ of the flight. If one adds all time periods during which the gondola/telescope was pointing within $\pm 26^{\prime \prime}$ for at least 1 min continuously, the total sum is over $72 \mathrm{~h}$, or $60 \%$ of the flight.

Long periods of continuous good pointing were needed for scientific goals, e.g., long exposures, calibrations and movies. Thus continuous, long duration pointing periods of accuracy better than $\pm 26^{\prime \prime}$ were highly desired. Table 4 shows the total sum of time over the entire flight that the gondola/ telescope was pointed continuously within $\pm 26^{\prime \prime}$ of the target for time periods of $30 \mathrm{~s}, 1 \mathrm{~min}, 2 \mathrm{~min}$, $5 \mathrm{~min}$ and $10 \mathrm{~min}$.

Shown in the fourth column of Table 4 are the statistics from the 2009 flight of Sunrise (Sunrise I).
Comparison shows Sunrise II pointing statistics were a factor of two better than Sunrise I. During Sunrise I, thumbnails were not available, so servo engineers on the ground had very limited information to fine tune the servos. Having the benefit of thumbnails and the ability to upload informed servo settings were pivotal in the improved performance of the 2013 flight.

Figure 10 shows the histogram of the durations of all good pointing periods in the 2013 flight. There were several time periods greater than an hour where the gondola/telescope was continually pointing within $\pm 26^{\prime \prime}$ of the target. Notably, a $99 \mathrm{~min}$ period of continuous good pointing was achieved.

Scientific objectives required re-pointing of the gondola/telescope for geometric scaling, flat fielding and targeting of interesting solar features. To save

\section{Sunrise II}

20130612 _110000 to 20130617_103009 entire flight

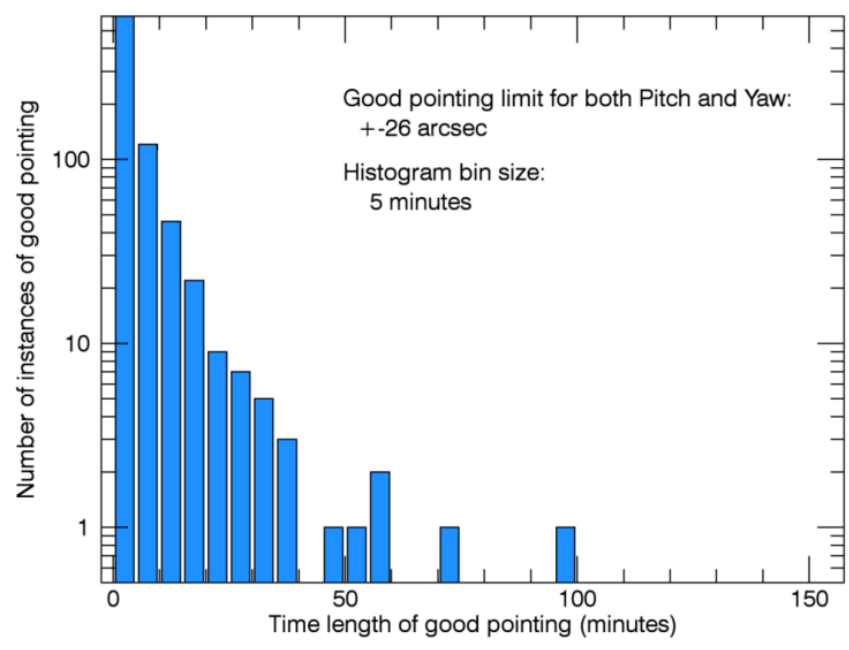

Fig. 10. Histogram of the length of periods of continuous pointing within $\pm 26^{\prime \prime}$ of the target during the 2013 flight of Sunrise. 
Sunrise II

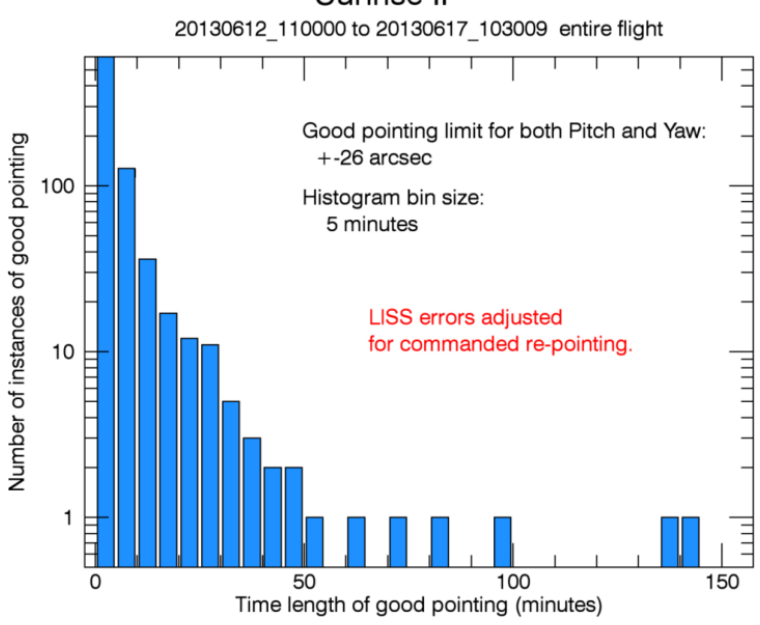

Fig. 11. Recomputed histogram of the length of periods of continuous pointing within $\pm 26^{\prime \prime}$ of the target during the 2013 flight of Sunrise after compensating for commanded re-pointing.

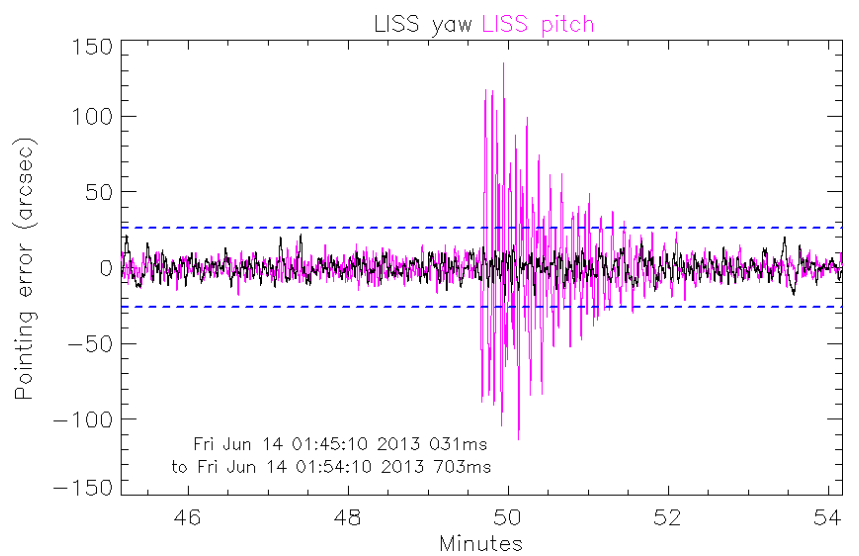

Fig. 12. Termination of a good pointing period due to elevation failure. The dashed blue lines in the LISS plot are visual aides showing the boundries of the $\pm 26^{\prime \prime}$ pointing limit.
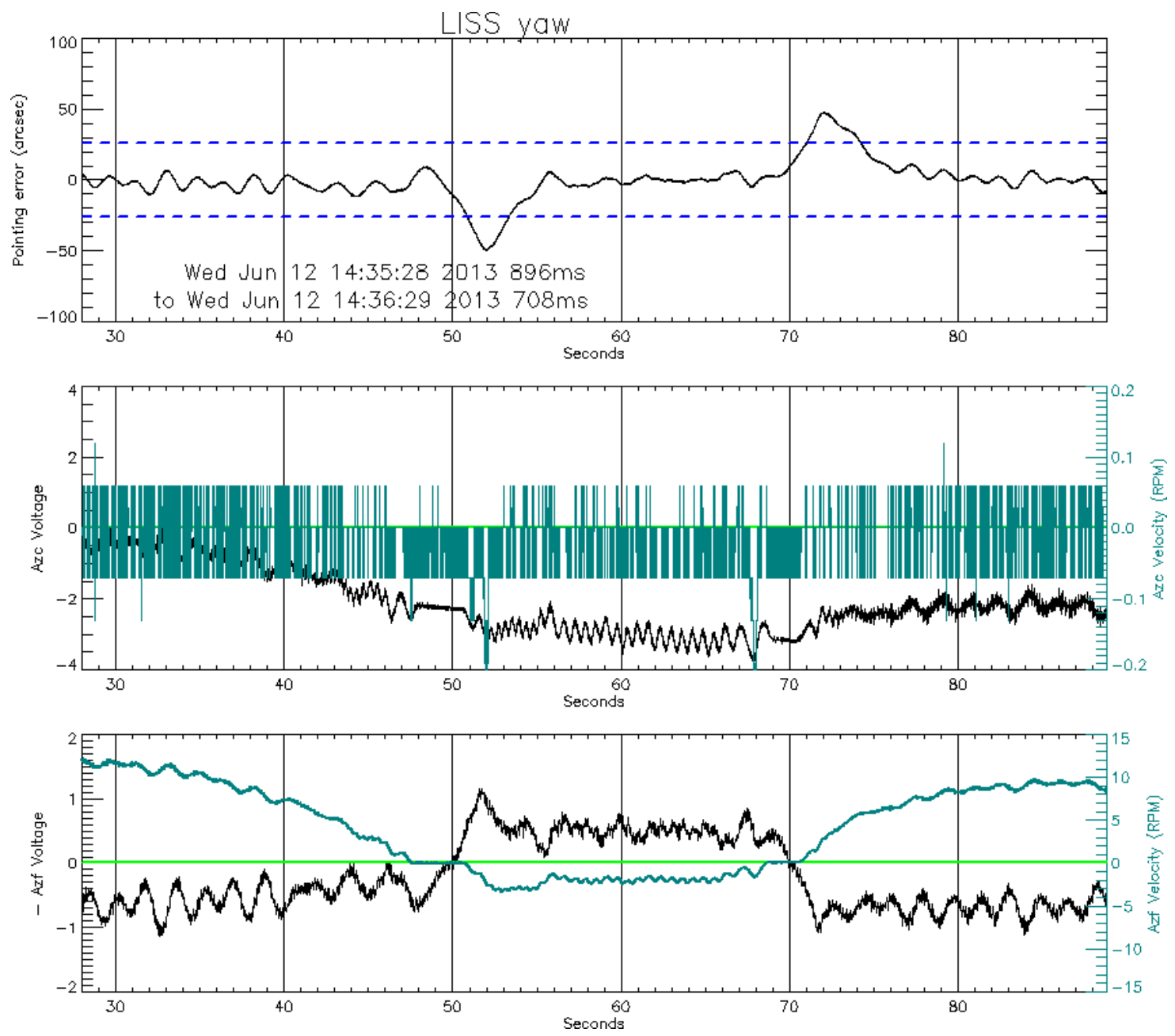

Fig. 13. Pointing failure due to stiction effects of the Azf reaction wheel. Stiction effects in the Azc motor are also evident. The dashed blue lines in the LISS plot are visual aides showing the boundries of the $\pm 26^{\prime \prime}$ pointing limit. The bright green lilne in each voltage/velocity plot is a visual aide marking zero voltage and velocity. 
time, re-pointing was done quickly and these sudden offsets disrupted the continuity of the good pointing. Without these perturbations, the PS could have attained longer periods of continuous good pointing. To test this hypothesis, the statistics for long periods of good pointing were rerun by setting all LISS errors to zero for $30 \mathrm{~s}$ after any re-pointing command was received. The re-computed histogram is shown in Fig. 11.

As can be seen from Fig. 11, adjusting for commanded re-pointing has combined several of the shorter periods together. Remarkably, two periods of good pointing exceeded 135 min. In comparison with the 2009 flight, as reported by Barthol et al. (2010), the longest period with telescope pointing within $\pm 46^{\prime \prime}$ of the target was $45 \mathrm{~min}$.

Termination of many good pointing periods resulted from elevation pointing failure, an example of which is shown in Fig. 12. Since these failures were sporadic events, thumbnails were not available for engineers to diagnose the problem. Post flight analysis revealed the failure was due to the very high $100 \mathrm{~Hz}$ frequency setting used for the integrator filter in the El_Local servo suite. Reducing the frequency by a few percent would likely eliminate the failure and still maintain pointing performance.

Occasionally, azimuthal pointing failure occurred as well. The AzfTrack servo did not include a friction compensation function. Thus, under circumstances where the velocity of the reaction wheel approached zero, overshoot/undershoot of the Azf motor voltage occurred. An example is given in Fig. 13. Around 14:35:32, a typical atmospheric disturbance perturbed the pointing and the servo required the reaction wheel to slow. Around 14:35:46 the velocity called for by the servo was positive, but very close to zero. However, friction caused the reaction wheel to stall and the pointing to further degrade. Larger LISS yaw error signals caused the servos to demand more voltage, beginning the overshoot/undershoot pattern.

The figure also shows that the Azc motor also suffered overshoot/undershoot stiction effects. The Azc velocity excursions that occurred at 14:35:52 and 14:36:08 were quite large, but produced by relatively small voltage changes.

This type of pointing problem did not occur frequently since the Azf motor was nearly always moving. But the easy addition of friction compensation functions to the output of the AzfTrack and
AzfToAzc servos could completely eliminate this failure mode.

\section{Conclusions}

The Sunrise II pointing system was able to maintain stable pointing within the required $\pm 26^{\prime \prime}$ of the target for a substantial portion of the 2013 flight. With this success, ISLiD and CWS were able to perform well, and the science instruments, IMaX and SUFI were able to obtain high quality data. Using Sunrise II observations, Riethmüller et al. (2013) and Danilovic et al. (2014) have presented the first high resolution images of quiet and active regions of the sun in the $\mathrm{Mg}$ II k $2796 \AA$ line. An upcoming special issue of The Astrophysical Journal (Supplement Series) will be devoted to Sunrise II and describe the many exciting results stemming from its observations.

Were a third flight of Sunrise to occur, easily made improvements to the PS, e.g., El_Local filter adjustments and friction compensation functions for the fine (AzfTrack) and coarse azimuth (AzfToAzc) servos, would provide even better pointing and longer duration times of continuous good pointing.

\section{Acknowledgments}

This work was performed under NASA grant number NNX13AE95G. The authors thank the talented staff of NCAR's Earth Observing Laboratory, Design and Fabrication Services. Their contributions to the pre-flight and post-flight efforts are greatly appreciated. Additionally, the authors wish to thank Peter G. Nelson and especially Clemens Halbgewachs who worked tirelessly in determining optimal servo settings. The authors are very grateful to Piyush Agrawal, Justus Brosche, Rebecca Centeno, Courtney Peck, and Jack Fox. Their wonderful help was invaluable in during PS set up and flight operations. The Sunrise II flight would not have been a success without the expertise of the dedicated staff of CSBF, ESRANGE, MPS, KIS, and IMaX. We thank them. The National Center for Atmospheric Research is sponsored by the National Science Foundation.

\section{References}

Barthol, P., Gandorfer, A., Solanki, S. K. et al. [2010], "The sunrise mission," Solar Phys. 268, 1, doi: 10.1007/s11207010-9662-9. 
Berkefeld, T., Schmidt, W., Soltau, D. et al. [2011] Solar Phys. 268, 103, doi: 10.1007/s11207-010-9676-3.

Danilovic, S., Hirzberger, J., Riethmüller, T. L. et al. [2014], ApJ 784, 20, doi: 10.1088/0004-637X/784/1/20.

Gandorfer, A., Grauf, B., Barthol, P. et al. [2011] Solar Phys. 268, 35, doi: 10.1007/s11207-010-9636-y.

Martínez Pillet, V., del Toro Iniesta, J. C., Álvarez-Herrero, A. et al. [2011] Solar Phys. 268, 57, doi: 10.1007/s11207-010-9644-y.
Pascale, E., Ade, P. A. R., Bock, J. J. et al. [2008] "The balloonborne large aperture submillimeter telescope: BLAST," ApJ 681, 400 .

Riethmüller, T. L., Solanki, S. K., Hirzberger, J. et al. [2013], ApJL 776, L13, doi: 10.1088/2041-8205/776/1/L13. 